\title{
Improved Approximation Algorithms for Metric Max TSP
}

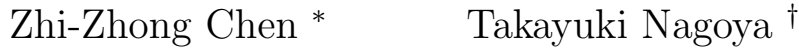

\begin{abstract}
We present two polynomial-time approximation algorithms for the metric case of the maximum traveling salesman problem. One of them is for directed graphs and its approximation ratio is $\frac{27}{35}$. The other is for undirected graphs and its approximation ratio is $\frac{7}{8}-o(1)$. Both algorithms improve on the previous bests.
\end{abstract}

\section{Introduction}

The maximum traveling salesman problem (MaxTSP) is to compute a maximum-weight Hamiltonian circuit (called a tour) in a given complete edge-weighted (undirected or directed) graph. Usually, MaxTSP is divided into the symmetric and the asymmetric cases. In the symmetric case, the input graph is undirected; we denote this case by SymMaxTSP. In the asymmetric case, the input graph is directed; we denote this case by AsymMaxTSP. Note that SymMaxTSP can be trivially reduced to AsymMaxTSP.

A natural constraint one can put on AsymMaxTSP and SymMaxTSP is the triangle inequality which requires that for every set of three vertices $u_{1}, u_{2}$, and $u_{3}$ in the input graph $G, w\left(u_{1}, u_{2}\right) \leq$ $w\left(u_{1}, u_{3}\right)+w\left(u_{3}, u_{2}\right)$, where $w\left(u_{i}, u_{j}\right)$ is the weight of the edge from $u_{i}$ to $u_{j}$ in $G$. If we put this constraint on AsymMaxTSP, we obtain a problem called metric AsymMaxTSP. Similary, if we put this constraint on SymMaxTSP, we obtain a problem called metric SymMaxTSP.

Both metric SymMaxTSP and metric AsymMaxTSP are Max-SNP-hard [1] and there have been a number of approximation algorithms known for them $[7,4,5]$. In 1985, Kostochka and Serdyukov [7] gave an $O\left(n^{3}\right)$-time approximation algorithm for metric SymMaxTSP that achieves an approximation ratio of $\frac{5}{6}$. Their algorithm is very simple and elegant. Tempted by improving the ratio $\frac{5}{6}$, Hassin and Rubinstein [4] gave a randomized $O\left(n^{3}\right)$-time approximation algorithm for metric SymMaxTSP whose expected approximation ratio is $\frac{7}{8}-o(1)$. This randomized algorithm was recently (partially) derandomized by Chen et al. [3]; their result is a (deterministic) $O\left(n^{3}\right)$-time approximation algorithm for metric SymMaxTSP whose approximation ratio is $\frac{17}{20}-o(1)$. In this paper, we completely derandomize the randomized algorithm, i.e., we obtain a (deterministic) $O\left(n^{3}\right)$-time approximation algorithm for metric SymMaxTSP whose approximation ratio is $\frac{7}{8}-o(1)$. Our algorithm also has the advantage of being easy to parallelize. Our derandomization is based on the idea of Chen et al. [3] and newly discovered properties of a folklore partition of the edges of a $2 n$-vertex complete undirected graph into $2 n-1$ perfect matchings. These properties may be useful elsewhere. In particular, one of the properties says that if $G=(V, E)$ is a $2 n$-vertex complete undirected graph and $M$ is a perfect matching of $G$, then we can partition $E-M$ into $2 n-2$ perfect matchings $M_{1}, \ldots, M_{2 n-2}$ among which there are at most $k^{2}-k$ perfect matchings $M_{i}$ such that the graph $\left(V, M \cup M_{i}\right)$ has a cycle of length at most $2 k$ for every natural number $k$. This property is interesting because Hassin and Rubinstein [4] prove that if $G$ and $M$ are as before and $M^{\prime}$ is a random perfect matching of $G$, then with probability $1-o(1)$ the multigraph $\left(V, M \cup M^{\prime}\right)$ has no cycle of length at most $\sqrt{n}$. Our result shows that instead

\footnotetext{
${ }^{*}$ Supported in part by the Grant-in-Aid for Scientific Research of the Ministry of Education, Science, Sports and Culture of Japan, under Grant No. 14580390. Department of Mathematical Sciences, Tokyo Denki University, Hatoyama, Saitama 350-0394, Japan. Email: chen@r.dendai.ac.jp.

${ }^{\dagger}$ Department of Mathematical Sciences, Tokyo Denki University, Hatoyama, Saitama 350-0394, Japan. Email: nagoya@r.dendai.ac.jp.
} 
of sampling from the set of all perfect matchings of $G$, it suffices to sample from $M_{1}, \ldots, M_{2 n-2}$. This enables us to completely derandomize their algorithm.

As for metric AsymMaxTSP, Kostochka and Serdyukov [7] gave an $O\left(n^{3}\right)$-time approximation algorithm that achieves an approximation ratio of $\frac{3}{4}$. Their result remained the best in two decades until Kaplan et al. [5] gave a polynomial-time approximation algorithm whose approximation ratio is $\frac{10}{13}$. The key in their algorithm is a polynomial-time algorithm for computing two cycle covers $\mathcal{C}_{1}$ and $\mathcal{C}_{2}$ in the input graph $G$ such that $\mathcal{C}_{1}$ and $\mathcal{C}_{2}$ do not share a 2-cycle and the sum of their weights is at least twice the optimal weight of a tour of $G$. They then observe that the multigraph formed by the edges in 2-cycles in $\mathcal{C}_{1}$ and $\mathcal{C}_{2}$ can be split into two subtours of $G$. In this paper, we show that the multigraph formed by the edges in 2-cycles in $\mathcal{C}_{1}$ and $\mathcal{C}_{2}$ together with a constant fraction of the edges in non-2-cycles in $\mathcal{C}_{1}$ and $\mathcal{C}_{2}$ can be split into two subtours of $G$. This enables us to improve Kaplan et al.'s algorithm to a polynomial-time approximation algorithm whose approximation ratio is $\frac{27}{35}$.

\section{Basic Definitions}

Throughout this paper, a graph means a simple undirected or directed graph (i.e., it has neither multiple edges nor self-loops), while a multigraph may have multiple edges but no self-loops.

Let $G$ be a multigraph. We denote the vertex set of $G$ by $V(G)$, and denote the edge set of $G$ by $E(G)$. For a subset $F$ of $E(G), G-F$ denotes the graph obtained from $G$ by deleting the edges in $F$. Two edges of $G$ are adjacent if they share an endpoint.

Suppose $G$ is undirected. The degree of a vertex $v$ in $G$ is the number of edges incident to $v$ in $G$. A cycle in $G$ is a connected subgraph of $G$ in which each vertex is of degree 2. A cycle cover of $G$ is a subgraph $H$ of $G$ with $V(H)=V(G)$ in which each vertex is of degree 2. A matching of $G$ is a (possibly empty) set of pairwise nonadjacent edges of $G$. A perfect matching of $G$ is a matching $M$ of $G$ such that each vertex of $G$ is an endpoint of an edge in $M$.

Suppose $G$ is directed. The indegree of a vertex $v$ in $G$ is the number of edges entering $v$ in $G$, and the outdegree of $v$ in $G$ is the number of edges leaving $v$ in $G$. A cycle in $G$ is a connected subgraph of $G$ in which each vertex has indegree 1 and outdegree 1 . A cycle cover of $G$ is a subgraph $H$ of $G$ with $V(H)=V(G)$ in which each vertex has indegree 1 and outdegree 1. A 2-path-coloring of $G$ is a partition of $E(G)$ into two subsets $E_{1}$ and $E_{2}$ such that both graphs $\left(V(G), E_{1}\right)$ and $\left(V(G), E_{2}\right)$ are collections of vertex-disjoint paths. $G$ is 2-path-colorable if it has a 2-path-coloring.

Suppose $G$ is undirected or directed. A path in $G$ is either a single vertex of $G$ or a subgraph of $G$ that can be transformed to a cycle by adding a single (new) edge. The length of a cycle or path $C$ is the number of edges in $C$. A $k$-cycle is a cycle of length $k$. A $3^{+}$-cycle is a cycle of length at least 3 . A tour (also called a Hamiltonian cycle) of $G$ is a cycle $C$ of $G$ with $V(C)=V(G)$. A subtour of $G$ is a subgraph $H$ of $G$ which is a collection of vertex-disjoint paths.

A closed chain is a directed graph that can be obtained from an undirected $k$-cycle $C$ with $k \geq 3$ by replacing each edge $\{u, v\}$ of $C$ with the two directed edges $(u, v)$ and $(v, u)$. Similarly, an open chain is a directed graph that can be obtained from an undirected path $P$ by replacing each edge $\{u, v\}$ of $P$ with the two directed edges $(u, v)$ and $(v, u)$. An open chain is trivial if it is a single vertex. A chain is a closed or open chain. A partial chain is a subgraph of a chain.

For a graph $G$ and a weighting function $w$ mapping each edge $e$ of $G$ to a nonnegative real number $w(e)$, the weight of a subset $F$ of $E(G)$ is $w(F)=\sum_{e \in F} w(e)$, and the weight of a subgraph $H$ of $G$ is $w(H)=w(E(H))$.

\section{New Algorithm for Metric AsymMaxTSP}

Throughout this section, fix an instance $(G, w)$ of metric AsymMaxTSP, where $G$ is a complete directed graph and $w$ is a function mapping each edge $e$ of $G$ to a nonnegative real number $w(e)$.

Let $O P T$ be the weight of a maximum-weight tour in $G$. Our goal is to compute a tour in $G$ whose weight is large compared to OPT. We first review Kaplan et al.'s algorithm and define several notations on the way. 


\subsection{Kaplan et al.'s Algorithm}

The key in their algorithm is the following:

Theorem 3.1 [5] We can compute two cycle covers $\mathcal{C}_{1}, \mathcal{C}_{2}$ in $G$ in polynomial time that satisfy the following two conditions:

1. $\mathcal{C}_{1}$ and $\mathcal{C}_{2}$ do not share a 2-cycle. In other words, if $C$ is a 2-cycle in $\mathcal{C}_{1}$ (respectively, $\mathcal{C}_{2}$ ), then $\mathcal{C}_{2}$ (respectively, $\mathcal{C}_{1}$ ) does not contain at least one edge of $C$.

2. $w\left(\mathcal{C}_{1}\right)+w\left(\mathcal{C}_{2}\right) \geq 2 \cdot O P T$.

Let $G_{2}$ be the subgraph of $G$ such that $V\left(G_{2}\right)=V(G)$ and $E\left(G_{2}\right)$ consists of all edges in 2-cycles in $\mathcal{C}_{1}$ and/or $\mathcal{C}_{2}$. Then, $G_{2}$ is a collection of vertex-disjoint chains. For each closed chain $C$ in $G_{2}$, we can compute two edge-disjoint tours $T_{1}$ and $T_{2}$ (each of which is of length at least 3), modify $\mathcal{C}_{1}$ by substituting $T_{1}$ for the 2-cycles shared by $C$ and $\mathcal{C}_{1}$, modify $\mathcal{C}_{2}$ by substituting $T_{2}$ for the 2-cycles shared by $C$ and $\mathcal{C}_{2}$, and further delete $C$ from $G_{2}$. After this modification of $\mathcal{C}_{1}$ and $\mathcal{C}_{2}$, the two conditions in Theorem 3.1 still hold. So, we can assume that there is no closed chain in $G_{2}$.

For each $i \in\{1,2\}$, let $W_{i, 2}$ denote the total weight of 2-cycles in $\mathcal{C}_{i}$, and let $W_{i, 3}=w\left(\mathcal{C}_{i}\right)-W_{i, 2}$. For convenience, let $W_{2}=\frac{1}{2}\left(W_{1,2}+W_{2,2}\right)$ and $W_{3}=\frac{1}{2}\left(W_{1,3}+W_{2,3}\right)$. Then, by Condition 2 in Theorem 3.1, we have $W_{2}+W_{3} \geq O P T$. Moreover, using an idea in [7], Kaplan et al. observed the following:

Lemma 3.2 [5] We can use $\mathcal{C}_{1}$ and $\mathcal{C}_{2}$ to compute a tour $T$ of $G$ with $w(T) \geq \frac{3}{4} W_{2}+\frac{5}{6} W_{3}$ in polynomial time.

Since each nontrivial open chain has a 2-path-coloring, we can use $G_{2}$ to compute a tour $T^{\prime}$ of $G$ with $w\left(T^{\prime}\right) \geq W_{2}$ in polynomial time. Combining this observation, Lemma 3.2, and the fact that $W_{2}+W_{3} \geq O P T$, the heavier one between $T$ and $T^{\prime}$ is of weight at least $\frac{10}{13} O P T$.

\subsection{Details of the New Algorithm}

The idea behind our new algorithm is to improve the second tour $T^{\prime}$ in Kaplan et al.'s algorithm so that it has weight at least $W_{2}+\frac{1}{9} W_{3}$. The tactics is to add some edges of $3^{+}$-cycles in $\mathcal{C}_{i}$ with $W_{i, 3}=\max \left\{W_{1,3}, W_{2,3}\right\}$ to $G_{2}$ so that $G_{2}$ remains 2-path-colorable. Without loss of generality, we may assume that $W_{1,3} \geq W_{2,3}$. Then, our goal is to add some edges of $3^{+}$-cycles in $\mathcal{C}_{1}$ to $G_{2}$ so that $G_{2}$ remains 2-path-colorable.

We say that an open chain $P$ in $G_{2}$ spoils an edge $(u, v)$ of a $3^{+}$-cycle in $\mathcal{C}_{1}$ if $u$ and $v$ are the two endpoints of $P$. Obviously, adding a spoiled edge to $G_{2}$ destroys the 2-path-colorability of $G_{2}$. Fortunately, there is no $3^{+}$-cycle in $\mathcal{C}_{1}$ in which two consecutive edges are both spoiled. So, let $C_{1}$, $\ldots, C_{\ell}$ be the $3^{+}$-cycles in $\mathcal{C}_{1}$; we modify each $C_{j}(1 \leq j \leq \ell$ ) as follows (see Figure 1 ):

- For every two consecutive edges $(u, v)$ and $(v, x)$ of $C_{j}$ such that $(u, v)$ is spoiled, replace $(u, v)$ by the two edges $(u, x)$ and $(x, v)$. (Comment: We call $(u, x)$ a bypass edge of $C_{j}$, call the 2 -cycle between $v$ and $x$ a dangling 2-cycle of $C_{j}$, and call $v$ the articulation vertex of the dangling 2-cycle. We also say that the bypass edge $(u, x)$ and the dangling 2-cycle between $v$ and $x$ correspond to each other.)

We call the above modification of $C_{j}$ the bypass operation on $C_{j}$. Note that applying the bypass operation on $C_{j}$ does not decrease the weight of $C_{j}$ because of the triangle inequality. Moreover, the edges of $C_{j}$ not contained in dangling 2-cycles of $C_{j}$ form a cycle. We call it the primary cycle of $C_{j}$. Note that $C_{j}$ may have neither bypass edges nor dangling 2-cycles (this happens when $C_{j}$ has no spoiled edges). 


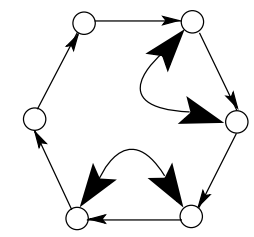

(1)

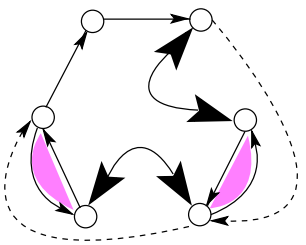

(2)

Figure 1: (1) A $3^{+}$-cycle $C_{j}$ (formed by the one-way edges) in $\mathcal{C}_{1}$ and the open chains (each shown by a two-way edge) each of which has a parallel edge in $C_{j}$. (2) The modified $C_{j}$ (formed by the one-way edges), where bypass edges are dashed and dangling 2-cycles are painted.

Let $H$ be the union of the modified $C_{1}, \ldots, C_{\ell}$, i.e., let $H$ be the directed graph with $V(H)=$ $\bigcup_{1 \leq j \leq \ell} V\left(C_{j}\right)$ and $E(H)=\bigcup_{1 \leq j \leq \ell} E\left(C_{j}\right)$. We next show that $E(H)$ can be partitioned into three subsets each of which can be added to $G_{2}$ without destroying its 2-path-colorability. Before proceeding to the details of the partitioning, we need several definitions and lemmas.

Two edges $\left(u_{1}, u_{2}\right)$ and $\left(v_{1}, v_{2}\right)$ of $H$ form a critical pair if $u_{1}$ and $v_{2}$ are the endpoints of some open chain in $G_{2}$ and $u_{2}$ and $v_{1}$ are the endpoints of another open chain in $G_{2}$ (see Figure 2). Note that adding both $\left(u_{1}, u_{2}\right)$ and $\left(v_{1}, v_{2}\right)$ to $G_{2}$ destroys its 2-path-colorability. An edge of $H$ is critical if it together with another edge of $H$ forms a critical pair. Note that for each critical edge $e$ of $H$, there is a unique edge $e^{\prime}$ in $H$ such that $e$ and $e^{\prime}$ form a critical pair. We call $e^{\prime}$ the rival of $e$. An edge of $H$ is safe if it is not critical. A bypass edge of $H$ is a bypass edge of a $C_{j}$ with $1 \leq j \leq \ell$. Similarly, a dangling 2-cycle of $H$ is a dangling 2-cycle of a $C_{j}$ with $1 \leq j \leq \ell$. A dangling edge of $H$ is an edge in a dangling 2-cycle of $H$.

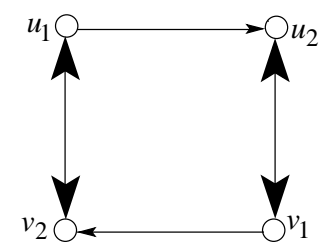

Figure 2: A critical pair formed by edges $\left(u_{1}, u_{2}\right)$ and $\left(v_{1}, v_{2}\right)$.

Lemma 3.3 No bypass edge of $H$ is critical.

Proof. Suppose that $e=\left(u_{1}, u_{2}\right)$ is a bypass edge of a $C_{j}$ with $1 \leq j \leq \ell$. Then, $u_{2}$ is the articulation vertex of a dangling 2-cycle $C$ of $C_{j}$. Let $u_{3}$ be the vertex of $C$ other than $u_{2}$. Then, there is an open chain $P$ in $G_{2}$ whose endpoints are $u_{1}$ and $u_{3}$. Since $e$ leaves $u_{1}$ and $e^{\prime}=\left(u_{2}, u_{3}\right)$ is the unique edge in $C_{j}$ entering $u_{3}, e^{\prime}$ has to be the rival of $e$ whenever $e$ is critical. However, by the definition of criticalness, each critical edge and its rival should not be adjacent. So, $e$ cannot be critical.

Lemma 3.4 Fix a $j$ with $1 \leq j \leq \ell$. Suppose that an edge e of $C_{j}$ is a critical dangling edge of $H$. Let $C$ be the dangling 2-cycle of $C_{j}$ containing $e$. Let $e^{\prime}$ be the rival of e. Then, the following statements hold:

1. $e^{\prime}$ is also an edge of $C_{j}$.

2. If $e^{\prime}$ is also a dangling edge of $H$, then the primary cycle of $C_{j}$ consists of the two bypass edges corresponding to $C$ and $C^{\prime}$, where $C^{\prime}$ is the dangling 2-cycle of $C_{j}$ containing $e^{\prime}$.

3. If $e^{\prime}$ is not a dangling edge of $H$, then $e^{\prime}$ is the edge in the primary cycle of $C_{j}$ whose head is the tail of the bypass edge corresponding to $C$.

Proof. Let $u_{1}$ be the articulation vertex of $C$, and let $u_{2}$ be the other vertex of $C$. Then, there is an open chain $P$ one of whose endpoints is $u_{2}$. Let $u_{3}$ be the other endpoint of $P$. We now prove the statements separately as follows. 
Statement 1. Note that $u_{3}$ must be a vertex of $C_{j}$ (indeed, $\left(u_{3}, u_{1}\right)$ is a bypass edge of $C_{j}$ ). By the definition of criticalness, the rival of $e$ is an edge incident to $u_{3}$. However, every edge of $H$ incident to $u_{3}$ is in $C_{j}$. Thus, the rival of $e$ must be in $C_{j}$ whenever $e$ is critical.

Statement 2. Suppose that $e^{\prime}$ is also a dangling edge of $H$. Then, since $e^{\prime}$ is incident to $u_{3}$ (as observed in the proof of Statement 1) and $u_{3}$ appears in the primary cycle of $C_{j}, u_{3}$ must be the articulation vertex of the dangling 2-cycle $C^{\prime}$ containing $e^{\prime}$. Let $u_{4}$ be the vertex of $C^{\prime}$ other than $u_{3}$. Then, by the definition of criticalness, there is an open chain in $G_{2}$ whose endpoints are $u_{4}$ and $u_{1}$. Now, $\left(u_{1}, u_{3}\right)$ has to be the bypass edge corresponding to $C^{\prime}$. Recall that $\left(u_{3}, u_{1}\right)$ is the bypass edge corresponding to $C$. This completes the proof of Statement 2.

Statement 3. Suppose that $e^{\prime}$ is not a dangling edge of $H$. Recall that $e^{\prime}$ is incident to $u_{3}$ and $\left(u_{3}, u_{1}\right)$ is a bypass edge of $C_{j}$. By Lemma $3.3, e^{\prime}$ cannot be $\left(u_{3}, u_{1}\right)$. So, $e^{\prime}$ has to be the edge in the primary cycle of $C_{j}$ entering $u_{3}$.

Lemma 3.5 Fix a $j$ with $1 \leq j \leq \ell$ such that the primary cycle $C$ of $C_{j}$ contains no bypass edge. Let $u_{1}, \ldots, u_{k}$ be a cyclic ordering of the vertices in $C$. Then, the following hold:

1. Suppose that there is a chain $P$ in $G_{2}$ whose endpoints appear in $C$ but not consecutively (i.e., its endpoints are not connected by an edge of $C$ ). Then, at least one edge of $C$ is safe.

2. Suppose that every edge of $C$ is critical. Then, there is a unique $C_{j^{\prime}}$ with $j^{\prime} \in\{1, \ldots, \ell\}-\{j\}$ such that (1) the primary cycle $C^{\prime}$ of $C_{j^{\prime}}$ has exactly $k$ vertices and (2) the vertices of $C^{\prime}$ have a cyclic ordering $v_{1}, \ldots, v_{k}$ such that for every $1 \leq i \leq k, u_{i}$ and $v_{k-i+1}$ are the endpoints of some chain in $G_{2}$. (See Figure 4.)

Proof. We prove the two statements separately as follows.

Statement 1. By the existence of $P$, we can find two vertices $u_{i}$ and $u_{h}$ in $C$ with $i<h$ such that (1) neither $\left(u_{i}, u_{h}\right)$ nor $\left(u_{h}, u_{i}\right)$ is an edge of $C,(2)$ there is a chain in $G_{2}$ whose endpoints are $u_{i}$ and $u_{h}$, and (3) there is no chain in $G_{2}$ whose endpoints both are in the set $\left\{u_{i+1}, u_{i+2}, \ldots, u_{h-1}\right\}$. Obviously, $\left(u_{i}, u_{i+1}\right)$ is safe.

Statement 2. Each vertex $u_{i}$ of $C$ is an endpoint of a chain $P_{i}$ in $G_{2}$ or else the two edges incident to $u_{i}$ would be safe. Moreover, $P_{1} \neq P_{2}, P_{2} \neq P_{3}, \ldots, P_{k-1} \neq P_{k}$, and $P_{k} \neq P_{1}$ because we have applied the bypass operation on $C_{j}$. Furthermore, by Statement 1 , there do not exist $i$ and $h$ with $1 \leq i \neq h \leq k$ with $P_{i}=P_{h}$. Therefore, for every $i \in\{1, \ldots, k\}$, the endpoint of $P_{i}$ other than $u_{i}$ is not in $C$.

For each $i \in\{1, \ldots, k\}$, let $v_{k-i+1}$ be the endpoint of $P_{i}$ other than $u_{i}$. Obviously, for each $i \in\{1, \ldots, k-1\},\left(v_{k-i}, v_{k-i+1}\right)$ has to be an edge of $H$ because $\left(u_{i}, u_{i+1}\right)$ is a critical edge. Similarly, $\left(v_{k}, v_{1}\right)$ has to be an edge of $H$ because $\left(u_{k}, u_{1}\right)$ is a critical edge. So, $v_{1}, \ldots, v_{k}$ is a cyclic ordering of the vertices of some cycle $C^{\prime}$ in $H$. Let $j^{\prime}$ be the integer in $\{1, \ldots, \ell\}$ such that $C^{\prime}$ is a cycle in $C_{j^{\prime}}$.

It remains to show that $C^{\prime}$ is not a dangling 2-cycle of $C_{j^{\prime}}$. For a contradiction, assume that $C^{\prime}$ is a dangling 2-cycle of $C_{j^{\prime}}$. Then, by Statement 1 in Lemma 3.4, $j=j^{\prime}$ and $C$ has to be the primary cycle of $C_{j^{\prime}}$. Moreover, since $C^{\prime}$ is a 2 -cycle, $C$ is a 2-cycle, too. But then, $\left\{u_{1}, u_{2}\right\} \cap\left\{v_{1}, v_{2}\right\} \neq \emptyset$, because the articulation vertex of $C^{\prime}$ has to be a vertex of $C$. This contradicts the fact that for each $i \in\{1, \ldots, k\}$, the endpoint of $P_{i}$ other than $u_{i}$ is not in $C$ (as observed above).

Now we are ready to describe how to partition $E(H)$ into three subsets each of which can be added to $G_{2}$ without destroying its 2-path-colorability. We use the three colors 0,1 , and 2 to represent the three subsets, and want to assign each edge of $E(H)$ a color in $\{0,1,2\}$ so that the following conditions are satisfied:

(C1) For every critical edge $e$ of $H, e$ and its rival receive different colors.

(C2) For every dangling 2-cycle $C$ of $H$, the two edges in $C$ receive the same color.

(C3) If two adjacent edges of $H$ receive the same color, then they form a 2-cycle of $H$. 
To compute a coloring of the edges of $H$ satisfying the above three conditions, we process $C_{1}, \ldots$, $C_{\ell}$ in an arbitrary order. While processing $C_{j}(1 \leq j \leq \ell)$, we color the edges of $C_{j}$ by distinguishing four cases as follows (where $C$ denotes the primary cycle of $C_{j}$ ):

Case 1: $C$ is a 2-cycle. Then, $C$ contains either one or two bypass edges. In the former (respectively, latter) case, we color the edges of $C_{j}$ as shown in Figure 3(2) (respectively, Figure 3(1)). Note that the colored edges satisfy Conditions (C1) through (C3) above.

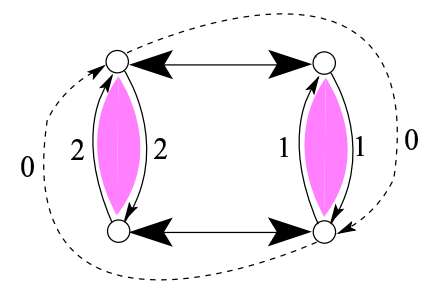

(1)

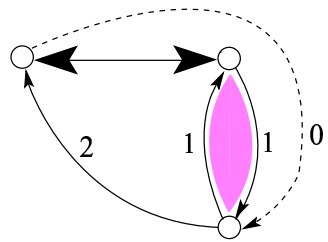

(2)

Figure 3: Coloring $C_{j}$ when its primary cycle is a 2-cycle.

Case 2: Every edge of $C$ is critical. Then, by Lemma 3.3, $C$ contains no bypass edge. Let $j^{\prime}$ be the integer in $\{1, \ldots, \ell\}-\{j\}$ such that $C_{j^{\prime}}$ satisfies the two conditions (1) and (2) in Statement 2 in Lemma 3.5. Then, by Lemma 3.4 and Statement 2 in Lemma 3.5, neither $C_{j}$ nor $C_{j^{\prime}}$ has a bypass edge or a dangling 2-cycle. So, the primary cycle of $C_{j}$ (respectively, $C_{j^{\prime}}$ ) is $C_{j}$ (respectively, $C_{j^{\prime}}$ ) itself. We color the edges of $C_{j}$ and $C_{j^{\prime}}$ simultaneously as follows (see Figure 4). First, we choose one edge $e$ of $C_{j}$, color $e$ with 2, and color the rival of $e$ with 0 . Note that the uncolored edges of $C_{j}$ form a path $Q$. Starting at one end of $Q$, we then color the edges of $Q$ alternatingly with colors 0 and 1 . Finally, for each uncolored edge $e^{\prime}$ of $C_{j^{\prime}}$, we color it with the color $h \in\{1,2\}$ such that the rival of $e^{\prime}$ has been colored with $h-1$. Note that the colored edges satisfy Conditions (C1) through (C3) above.

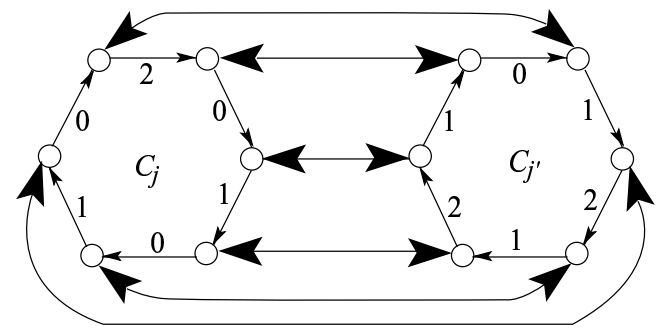

Figure 4: Coloring $C_{j}$ and $C_{j^{\prime}}$ when all their edges are critical.

Case 3: Neither Case 1 nor Case 2 occurs and no edge of $C_{j}$ is a critical dangling edge of $H$. Then, by Lemma 3.3 and Statement 1 in Lemma 3.5, $C$ contains at least one safe edge. Let $e_{1}, \ldots, e_{k}$ be the edges of $C$, and assume that they appear in $C$ cyclically in this order. Without loss of generality, we may assume that $e_{1}$ is a safe edge. We color $e_{1}$ with 0 , and then color the edges $e_{2}, \ldots, e_{k}$ in this order as follows. Suppose that we have just colored $e_{i}$ with a color $h_{i} \in\{0,1,2\}$ and we want to color $e_{i+1}$ next, where $1 \leq i \leq k-1$. If $e_{i+1}$ is a critical edge and its rival has been colored with $\left(h_{i}+1\right) \bmod 3$, then we color $e_{i+1}$ with $\left(h_{i}+2\right) \bmod 3$; otherwise, we color $e_{i+1}$ with $\left(h_{i}+1\right) \bmod 3$. If $e_{k}$ is colored 0 at the end, then we change the color of $e_{1}$ from 0 to the color in $\{1,2\}$ that is not the color of $e_{2}$. Now, we can further color each dangling 2-cycle $C^{\prime}$ of $C_{j}$ with the color in $\{0,1,2\}$ that has not been used to color the two edges of $C$ incident to the articulation vertex of $C^{\prime}$. Note that the colored edges satisfy Conditions (C1) through (C3) above.

Case 4: Neither Case 1 nor Case 2 occurs and some edge of $C_{j}$ is a critical dangling edge of $H$. For each dangling edge $e$ of $H$ with $e \in E\left(C_{j}\right)$, we define the partner of $e$ to be the edge $e^{\prime}$ of $C$ leaving the articulation vertex $u$ of the dangling 2-cycle containing $e$, and define the mate of $e$ to be the bypass edge $e^{\prime \prime}$ of $C_{j}$ entering $u$ (see Figure 6). We say that an edge $e$ of $C_{j}$ is bad if $e$ is a critical dangling edge of $H$ and its partner is the rival of another critical dangling edge of $H$. If $C_{j}$ has a bad edge $e$, then Statement 3 in Lemma 3.4 ensures that $C_{j}$ is as shown in Figure 5 and can be colored as shown there without violating Conditions (C1) through (C3) above. 


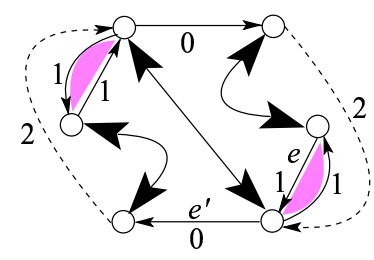

Figure 5: $C_{j}$ (formed by the one-way edges) and its coloring when it has a bad edge $e$.

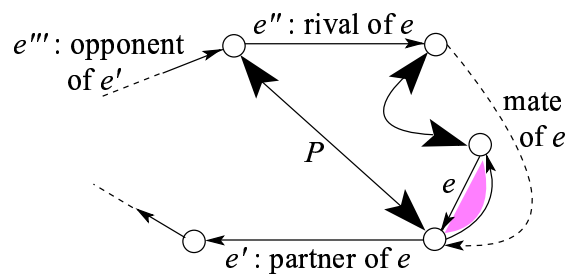

Figure 6: The rival, the mate, and the partner of a critical dangling edge $e$ of $H$ together with the opponent of the partner of $e$.

So, suppose that $C_{j}$ has no bad edge. We need one more definition (see Figure 6). Consider a critical dangling edge $e$ of $H$ with $e \in E\left(C_{j}\right)$. Let $e^{\prime}$ and $e^{\prime \prime}$ be the partner and the rival of $e$, respectively. Let $e^{\prime \prime \prime}$ be the edge of $C$ entering the tail of $e^{\prime \prime}$. Let $P$ be the open chain in $G_{2}$ whose endpoints are the tails of $e^{\prime}$ and $e^{\prime \prime}$. We call $e^{\prime \prime \prime}$ the opponent of $e^{\prime}$. Note that $e^{\prime} \neq e^{\prime \prime \prime}$ because the endpoints of $P$ are the tail of $e^{\prime}$ and the head of $e^{\prime \prime \prime}$. Moreover, if $e^{\prime}$ is a critical edge of $H$, then the rival of $e^{\prime}$ has to be $e^{\prime \prime \prime}$ because $e$ is not bad and $P$ exists. In other words, whenever an edge of $C$ has both its rival and its opponent, they must be the same. Similarly, if $e^{\prime \prime \prime}$ is a critical edge of $H$, then its rival has to be $e^{\prime}$. Obviously, neither $e^{\prime}$ nor $e^{\prime \prime \prime}$ can be the rival or the mate of a critical dangling edge of $H$ (because $C_{j}$ has no bad edge).

Now, let $e_{1}, \ldots, e_{q}$ be the edges of $C$ none of which is the rival or the mate of a critical dangling edge of $C_{j}$. We may assume that $e_{1}, \ldots, e_{q}$ appear in $C$ cyclically in this order. Without loss of generality, we may further assume that $e_{1}$ is the partner of a critical dangling edge of $H$. Then, we color $e_{1}$ with 0 , and further color $e_{2}, \ldots, e_{q}$ in this order as follows. Suppose that we have just colored $e_{i}$ with a color $h_{i} \in\{0,1,2\}$ and we want to color $e_{i+1}$ next, where $1 \leq i \leq q-1$. If $e_{i+1}$ is a critical edge of $H$ and its rival or opponent has been colored with $\left(h_{i}+1\right) \bmod 3$, then we color $e_{i+1}$ with $\left(h_{i}+2\right) \bmod 3$; otherwise, we color $e_{i+1}$ with $\left(h_{i}+1\right) \bmod 3$. Note that the colored edges satisfy Conditions (C1) through (C3) above, because the head of $e_{q}$ is not the tail of $e_{1}$.

We next show how to color the rival and the mate of each critical dangling edge of $C_{j}$. For each critical dangling edge $e$ of $C_{j}$, since its partner $e^{\prime}$ and the opponent of $e^{\prime}$ have been colored, we can color the rival of $e$ with the color of $e^{\prime}$ and color the mate of $e$ with a color in $\{0,1,2\}$ that is not the color of $e^{\prime}$. Note that the colored edges satisfy Conditions (C1) through (C3) above, because $e^{\prime}$ and its opponent have different colors.

Finally, for each dangling 2-cycle $D$ of $C_{j}$, we color the two edges of $D$ with the color in $\{0,1,2\}$ that has not been used to color an edge incident to the articulation vertex of $D$. Note that the colored edges satisfy Conditions (C1) through (C3) above, because the rival of each critical dangling edge $e$ of $H$ has the same color as the partner of $e$ does. This completes the coloring of $C_{j}$ (and hence $H$ ).

We next want to show how to use the coloring to find a large-weight tour in $G$. For each $i \in$ $\{0,1,2\}$, let $E_{i}$ be the edges of $H$ with color $i$. Without loss of generality, we may assume that $w\left(E_{0}\right) \geq \max \left\{w\left(E_{1}\right), w\left(E_{2}\right)\right\}$. Then, $w\left(E_{0}\right) \geq \frac{1}{3} W_{1,3}$ (see the beginning of this subsection for $W_{1,3}$ ). Consider the undirected graph $U=\left(V(G), F_{1} \cup F_{2}\right)$, where $F_{1}$ consists of all edges $\left\{v_{1}, v_{2}\right\}$ such that $\left(v_{1}, v_{2}\right)$ or $\left(v_{2}, v_{1}\right)$ is an edge in $E_{0}$, and $F_{2}$ consists of all edges $\left\{v_{3}, v_{4}\right\}$ such that $v_{3}$ and $v_{4}$ are the endpoints of an open chain in $G_{2}$. We further assign a weight to each edge of $F_{1}$ as follows. We first initialize the weight of each edge of $F_{1}$ to be 0 . For each edge $\left(v_{1}, v_{2}\right) \in E_{0}$, we then add the weight of edge $\left(v_{1}, v_{2}\right)$ to the weight of edge $\left\{v_{1}, v_{2}\right\}$. Note that for each $i \in\{1,2\}$, each connected component of the undirected graph $\left(V(G), F_{i}\right)$ is a single vertex or a single edge because of Condition (C3) above. So, each connected component of $U$ is a path or a cycle. Moreover, each cycle of $U$ contains at least 
three edges of $F_{1}$ because of Condition (C1) above. For eacy cycle $D$ of $U$, we mark exactly one edge $\left\{v_{1}, v_{2}\right\} \in F_{1}$ in $D$ whose weight is the smallest among all edges $\left\{v_{1}, v_{2}\right\} \in F_{1}$ in $D$. Let $E_{3}$ be the set of all edges $\left(v_{1}, v_{2}\right) \in E_{0}$ such that $\left\{v_{1}, v_{2}\right\}$ is marked. Then, $w\left(E_{3}\right) \leq \frac{1}{3} w\left(E_{0}\right)$. Consider the directed graph $G_{2}^{\prime}$ obtained from $G_{2}$ by adding the edges of $E_{0}-E_{3}$. Obviously, $w\left(G_{2}^{\prime}\right) \geq\left(W_{1,2}+W_{2,2}\right)+\frac{1}{9} W_{1,3}$. Moreover, $G_{2}^{\prime}$ is a collection of partial chains and hence is 2-path-colorable. So, we can partition the edges of $G_{2}^{\prime}$ into two subsets $E_{1}^{\prime}$ and $E_{2}^{\prime}$ such that both graphs $\left(V(G), E_{1}^{\prime}\right)$ and $\left(V(G), E_{2}^{\prime}\right)$ are subtours of $G$. The heavier one among the two subtours can be completed to a tour of $G$ of weight at least $\frac{1}{2}\left(W_{1,2}+W_{2,2}\right)+\frac{1}{18} W_{1,3} \geq W_{2}+\frac{1}{9} W_{3}$. Combining this with Lemma 3.2, we now have:

Theorem 3.6 There is a polynomial-time approximation algorithm for AsymMaxTSP achieving an approximation ratio of $\frac{27}{35}$.

\section{New Algorithm for Metric SymMaxTSP}

Throughout this section, fix an instance $(G, w)$ of metric SymMaxTSP, where $G$ is a complete undirected graph with $n$ vertices and $w$ is a function mapping each edge $e$ of $G$ to a nonnegative real number $w(e)$. Because of the triangle inequality, the following fact holds (see [3] for a proof):

Fact 4.1 Suppose that $P_{1}, \ldots, P_{t}$ are vertex-disjoint paths in $G$ each containing at least one edge. For each $1 \leq i \leq t$, let $u_{i}$ and $v_{i}$ be the endpoints of $P_{i}$. Then, we can use some edges of $G$ to connect $P_{1}, \ldots, P_{t}$ into a single cycle $C$ in linear time such that $w(C) \geq \sum_{i=1}^{t} w\left(P_{i}\right)+\frac{1}{2} \sum_{i=1}^{t} w\left(\left\{u_{i}, v_{i}\right\}\right)$.

Like Hassin and Rubinstein's algorithm (H\&R2-algorithm) for the problem, our algorithm computes two tours $T_{1}$ and $T_{2}$ of $G$ and outputs the one with the larger weight. The first two steps of our algorithm are the same as those of H\&R2-algorithm:

1. Compute a maximum-weight cycle cover $\mathcal{C}$. Let $C_{1}, \ldots, C_{r}$ be the cycles in $G$.

2. Compute a maximum-weight matching $M$ in $G$.

Lemma 4.2 [3] In linear time, we can compute two disjoint subsets $A_{1}$ and $A_{2}$ of $\bigcup_{1 \leq i \leq r} E\left(C_{i}\right)-M$ satisfying the following conditions:

(a) For each $j \in\{1,2\}$, each connected component of the graph $\left(V(G), M \cup A_{j}\right)$ is a path of length at least 1.

(b) For each $j \in\{1,2\}$ and each $i \in\{1, \ldots, r\},\left|A_{j} \cap E\left(C_{i}\right)\right|=1$.

For a technical reason, we will allow our algorithm to use only 1 random bit (so we can easily derandomize it, although we omit the details). The third through the seventh steps of our algorithm are as follows:

3. Compute two disjoint subsets $A_{1}$ and $A_{2}$ of $\bigcup_{1 \leq i \leq r} E\left(C_{i}\right)-M$ satisfying the two conditions in Lemma 4.2.

4. Choose $A$ from $A_{1}$ and $A_{2}$ uniformly at random.

5. Obtain a collection of vertex-disjoint paths each of length at least 1 by deleting the edges in $A$ from $\mathcal{C}$; and then connect these paths into a single (Hamiltonian) cycle $T_{1}$ as described in Fact 4.1.

6. Let $S=\{v \in V(G) \mid$ the degree of $v$ in the $\operatorname{graph}(V, M \cup A)$ is 1$\}$ and $F=\{\{u, v\} \in$ $E(G) \mid\{u, v\} \subseteq S\}$. Let $H$ be the complete graph $(S, F)$. Let $\ell=\frac{1}{2}|S|$. (Comment: $|S|$ is even, because of Condition (a) in Lemma 4.2.)

7. Let $M^{\prime}$ be the set of all edges $\{u, v\} \in F$ such that some connected component of the graph $\left(V, M \cup A\right.$ ) contains both $u$ and $v$. (Comment: $M^{\prime}$ is a perfect matching of $H$ because of Condition (a) in Lemma 4.2.)

Lemma 4.3 [3] Let $\alpha=w\left(A_{1} \cup A_{2}\right) / w(\mathcal{C})$. For a random variable $X$, let $\mathcal{E}[X]$ denote its expected value. Then, $\mathcal{E}[w(F)] \geq \frac{1}{4}(1-\alpha)(2 \ell-1) w(\mathcal{C})$. 
The next lemma shows that there cannot exist matchings of large weight in an edge-weighted graph where the weights satisfy the triangle inequality:

Lemma 4.4 For every perfect matching $N$ of $H, w(N) \leq w(F) / \ell$.

Proof. Let the edges of $N$ be $\left\{u_{1}, u_{2}\right\},\left\{u_{3}, u_{4}\right\}, \ldots,\left\{u_{2 \ell-1}, u_{2 \ell}\right\}$.

Case 1: $\ell$ is odd. For each odd number $i$ with $1 \leq i \leq \ell$, we assign the vertices $u_{i+2}, u_{i+3}$, $\ldots, u_{\ell+i}$ of $H$ to the edge $\left\{u_{i}, u_{i+1}\right\}$ of $N$. For each even number $j$ with $1 \leq j \leq \ell$, we assign the vertices $u_{1}, u_{2}, \ldots, u_{j}, u_{\ell+j+2}, u_{\ell+j+3}, \ldots, u_{2 \ell}$ of $H$ to the edge $\left\{u_{\ell+j}, u_{\ell+j+1}\right\}$ of $N$. Note that each edge in $N$ is assigned exactly $\ell-1$ vertices of $H$. For each edge $e_{i}=\left\{u_{i}, u_{i+1}\right\} \in N$ and each vertex $u_{h}$ assigned to $e_{i}$, we then assign the two edges $\left\{u_{i}, u_{h}\right\}$ and $\left\{u_{i+1}, u_{h}\right\}$ of $H$ to $e_{i}$. Since $w\left(\left\{u_{i}, u_{h}\right\}\right)+w\left(\left\{u_{i+1}, u_{h}\right\}\right) \geq w\left(e_{i}\right)$ by the triangle inequality, the total weight of edges assigned to each edge $e_{i} \in N$ is at least $(\ell-1) w\left(e_{i}\right)$. Obviously, no edge of $N$ is assigned to itself or another edge of $N$. Moreover, a simple but crucial observation is that no edge of $H$ is assigned to two or more edges of $N$. Thus, $w(F-N) \geq(\ell-1) w(N)$. Hence, $w(N) \leq w(F) / \ell$.

Case 2: $\ell$ is even. Let $N_{1}=\left\{\left\{u_{1}, u_{2}\right\},\left\{u_{3}, u_{4}\right\}, \ldots,\left\{u_{n-1}, u_{n}\right\}\right\}$ and $N_{2}=N-N_{1}$. We assume that $w\left(N_{1}\right) \geq w\left(N_{2}\right)$; the other case is similar. For each odd number $i$ with $1 \leq i \leq \ell-1$, we assign the vertices $u_{i+2}, u_{i+3}, \ldots, u_{\ell+i+1}$ of $H$ to the edge $\left\{u_{i}, u_{i+1}\right\}$ of $N$, and assign the vertices $u_{1}, u_{2}, \ldots, u_{i-1}, u_{\ell+i+2}, u_{\ell+i+3}, \ldots, u_{2 \ell}$ of $H$ to the edge $\left\{u_{\ell+i}, u_{\ell+i+1}\right\}$ of $N$. Note that each edge in $N_{1}$ (respectively, $N_{2}$ ) is assigned exactly $\ell$ (respectively, $\ell-2$ ) vertices of $H$. For each edge $e_{i}=\left\{u_{i}, u_{i+1}\right\} \in N$ and each vertex $u_{h}$ assigned to $e_{i}$, we then assign the two edges $\left\{u_{i}, u_{h}\right\}$ and $\left\{u_{i+1}, u_{h}\right\}$ of $H$ to $e_{i}$. Since $w\left(\left\{u_{i}, u_{h}\right\}\right)+w\left(\left\{u_{i+1}, u_{h}\right\}\right) \geq w\left(e_{i}\right)$ by the triangle inequality, the total weight of edges assigned to each edge $e_{i} \in N_{1}$ (respectively, $e_{i} \in N_{2}$ ) is at least $\ell w\left(e_{i}\right)$ (respectively, $\left.(\ell-2) w\left(e_{i}\right)\right)$. Obviously, no edge of $N$ is assigned to itself or another edge of $N$. Moreover, a simple but crucial observation is that no edge of $H$ is assigned to two or more edges of $N$. Thus, $w(F-N) \geq \ell w\left(N_{1}\right)+(\ell-2) w\left(N_{2}\right) \geq(\ell-1) w(N)$. Hence, $w(N) \leq w(F) / \ell$.

The following is our main lemma and will be proved in Section 4.1:

Lemma 4.5 We can partition $F-M^{\prime}$ into $2 \ell-2$ perfect matchings $M_{1}, \ldots, M_{2 \ell-2}$ of $H$ in linear time satisfying the following condition:

- For every natural number $q$, there are at most $q^{2}-q$ matchings $M_{i}$ with $1 \leq i \leq 2 \ell-2$ such that the graph $\left(S, M^{\prime} \cup M_{i}\right)$ has a cycle of length at most $2 q$.

Now, the eighth through the thirteenth steps of our algorithm are as follows:

8. Partition $F-M^{\prime}$ into $2 \ell-2$ perfect matchings $M_{1}, \ldots, M_{2 \ell-2}$ of $H$ in linear time satisfying the condition in Lemma 4.5.

9. Let $q=\lceil\sqrt[3]{\ell}\rceil$. Find a matching $M_{i}$ with $1 \leq i \leq 2 \ell-2$ satisfying the following two conditions:

(a) The graph $\left(S, M^{\prime} \cup M_{i}\right)$ has no cycle of length at most $2 q$.

(b) $w\left(M_{i}\right) \geq w\left(M_{j}\right)$ for all matchings $M_{j}$ with $1 \leq j \leq 2 \ell-2$ such that the graph $\left(S, M^{\prime} \cup M_{j}\right)$ has no cycle of length at most $2 q$.

10. Construct the graph $G_{i}^{\prime}=\left(V(G), M \cup A \cup M_{i}\right)$. (Comment: $M_{i} \cap(M \cup A)=\emptyset$ and each connected component of $G_{i}^{\prime}$ is either a path, or a cycle of length $2 q+1$ or more.)

11. For each cycle $D$ in $G_{i}^{\prime}$, mark exactly one edge $e \in M_{i} \cap E(D)$ such that $w(e) \leq w\left(e^{\prime}\right)$ for all $e^{\prime} \in M_{i} \cap E(D)$.

12. Obtain a collection of vertex-disjoint paths each of length at least 1 by deleting the marked edges from $G_{i}^{\prime}$; and then connect these paths into a single (Hamiltonian) cycle $T_{2}$ as described in Fact 4.1.

13. If $w\left(T_{1}\right) \geq w\left(T_{2}\right)$, output $T_{1}$; otherwise, output $T_{2}$.

Theorem 4.6 There is an $O\left(n^{3}\right)$-time approximation algorithm for metric SymMaxTSP achieving an approximation ratio of $\frac{7}{8}-O(1 / \sqrt[3]{n})$. 
Proof. Let $O P T$ be the maximum weight of a tour in $G$. It suffices to prove that $\max \left\{\mathcal{E}\left[w\left(T_{1}\right)\right]\right.$, $\left.\mathcal{E}\left[w\left(T_{2}\right)\right]\right\} \geq\left(\frac{7}{8}-O(1 / \sqrt[3]{n})\right) O P T$. By Fact 4.1, $\mathcal{E}\left[w\left(T_{1}\right)\right] \geq\left(1-\frac{1}{2} \alpha+\frac{1}{4} \alpha\right) w(\mathcal{C}) \geq\left(1-\frac{1}{4} \alpha\right) O P T$.

We claim that $|S| \geq \frac{1}{3} n$. To see this, consider the graphs $G_{M}=(V(G), M)$ and $G_{A}=(V(G), M \cup$ $A$ ). Because the length of each cycle in $\mathcal{C}$ is at least $3,|A| \leq \frac{1}{3} n$ by Condition (b) in Lemma 4.2. Moreover, since $M$ is a matching of $G$, the degree of each vertex in $G_{M}$ is 0 or 1 . Furthermore, $G_{A}$ is obtained by adding the edges of $A$ to $G_{M}$. Since adding one edge of $A$ to $G_{M}$ increases the degrees of at most two vertices, there exist at least $n-2|A| \geq \frac{1}{3} n$ vertices of degree 0 or 1 in $G_{A}$. So, by Condition (a) in Lemma 4.2, there are at least $\frac{1}{3} n$ vertices of degree 1 in $G_{A}$. This establishes that $|S| \geq \frac{1}{3} n$. Hence, $\ell \geq \frac{1}{6} n$.

Now, let $x$ be the number of matchings $M_{j}$ with $1 \leq j \leq 2 \ell-2$ such that the graph $\left(S, M^{\prime} \cup M_{i}\right)$ has a cycle of length at most $2 q$. Then, by Lemmas 4.4 and 4.5 , the weight of the matching $M_{i}$ found in Step 9 is at least $\left(1-\frac{x+1}{\ell}\right) \cdot w(F) \cdot \frac{1}{2 \ell-2-x}$. So, $w\left(M_{i}\right) \geq \frac{1}{\ell} \cdot\left(1-\frac{\ell-1}{2 \ell-2-q^{2}+q}\right) \cdot w(F)$ because $x \leq q^{2}-q$. Let $N_{i}$ be the set of edges of $M_{i}$ marked in Step 11. Then, $w\left(M_{i}-N_{i}\right) \geq \frac{q}{q+1} \cdot \frac{\ell-q^{2}+q-1}{\ell\left(2 \ell-2-q^{2}+q\right)} \cdot w(F)$. Hence, by Lemma 4.3 and the inequality $\ell \geq \frac{1}{6} n$, we have $\mathcal{E}\left[w\left(M_{i}-N_{i}\right)\right] \geq \frac{1}{4}(1-\alpha)(1-O(1 / \sqrt[3]{n})) w(\mathcal{C})$.

Obviously, $\mathcal{E}\left[w\left(T_{2}\right)\right] \geq \mathcal{E}[w(M \cup A)]+\mathcal{E}\left[w\left(M_{i}-N_{i}\right)\right] \geq\left(\frac{1}{2}-\frac{1}{2 n}\right) O P T+\frac{1}{2} \alpha w(\mathcal{C})+\mathcal{E}\left[w\left(M_{i}-\right.\right.$ $\left.\left.N_{i}\right)\right]$. Hence, by the last inequality in the previous paragraph, $\mathcal{E}\left[w\left(T_{2}\right)\right] \geq\left(\frac{3}{4}+\frac{1}{4} \alpha-O(1 / \sqrt[3]{n})\right) O P T$. Combining this with the inequality $\mathcal{E}\left[w\left(T_{1}\right)\right] \geq\left(1-\frac{1}{4} \alpha\right) O P T$, we finally have $\mathcal{E}\left[\max \left\{w\left(T_{1}\right), w\left(T_{2}\right)\right\}\right] \geq$ $\left(\frac{7}{8}-O(1 / \sqrt[3]{n})\right) O P T$.

The running time of the algorithm is dominated by the $O\left(n^{3}\right)$ time needed for computing a maximum-weight cycle cover and a maximum-weight matching.

As observed in [3], the subsets $A_{1}$ and $A_{2}$ in Lemma 4.2 can be computed in $O\left(\log ^{3} n\right)$ time using a linear number of processors. So, our algorithm for metric Max TSP is parallelizable because maximumweight cycle covers and maximum-weight matchings can be computed by fast parallel algorithms $[6,8]$. We omit the details here.

\subsection{Partitioning into Perfect Matchings}

Let the vertices of $H$ be $\infty, 0,1, \ldots, 2 \ell-2$, and let the edges of $M^{\prime}$ be

$$
\{\infty, 0\},\{1,2 \ell-2\},\{2,2 \ell-3\}, \ldots,\{\ell-1, \ell\} .
$$

Then, a folklore partitioning of $F-M^{\prime}$ into $2 \ell-2$ perfect matchings $M_{1}, \ldots, M_{2 \ell-2}$ of $H$ is as follows:

$$
\begin{gathered}
M_{1}:\{\infty, 1\},\{2,0\},\{3,2 \ell-2\}, \ldots,\{\ell, \ell+1\} \\
M_{2}:\{\infty, 2\},\{3,1\},\{4,0\}, \ldots,\{\ell+1, \ell+2\} \\
\vdots \\
M_{2 \ell-2}:\{\infty, 2 \ell-2\},\{0,2 \ell-3\},\{1,2 \ell-4\}, \ldots,\{\ell-2, \ell-1\} .
\end{gathered}
$$

For each integer $j \notin\{0,1, \ldots, 2 \ell-2\}$, we identify $j$ with the vertex $h$ of $H$ such that $h \equiv$ $j(\bmod 2 \ell-1)$. Then, for each integer $i \in\{0,1, \ldots, 2 \ell-2\}, M_{i}$ consists of edge $\{\infty, i\}$ and all edges $\{j,-j+2 i\}$ with $j \in\{0,1, \ldots, 2 \ell-2\}-\{i\}$. Obviously, for each $i \in\{1, \ldots, 2 \ell-2\}$, the graph $H_{i}=\left(S, M_{i} \cup M^{\prime}\right)$ is a collection of vertex-disjoint cycles; we call the cycle containing vertex $\infty$ the main cycle of $H_{i}$ and denote it by $D_{i}$. For two natural numbers $x$ and $y$, let $\operatorname{gcd}(x, y)$ denote the greatest common divisor of $x$ and $y$, and let $\operatorname{lcm}(x, y)$ denote the least common multiple of $x$ and $y$.

Lemma 4.7 For each $i \in\{1, \ldots, 2 \ell-2\}$, the length of $D_{i}$ is $\left(\frac{2 \ell-1}{\operatorname{gcd}(2 \ell-1, i)}+1\right)$.

Proof. Recall that for each integer $i \in\{0,1, \ldots, 2 \ell-2\}, M_{i}$ consists of edge $\{\infty, i\}$ and all edges $\{j,-j+2 i\}$ with $j \in\{0,1, \ldots, 2 \ell-2\}-\{i\}$. Fix an $i \in\{1, \ldots, 2 \ell-2\}$. Let $2 h$ be the length of $D_{i}$. 
Suppose that we traverse $D_{i}$ by starting at vertex $\infty$, then visiting $i$, and proceeding along the cycle until reaching vertex 0 . This traversal should give the following ordering of the vertices of $D_{i}$ :

$$
\infty, i,-i, 3 i,-3 i, 5 i, \cdots,-(2 h-3) i,(2 h-1) i
$$

where $(2 h-1) i \equiv 0(\bmod 2 \ell-1)$ because vertex 0 is the last one in the traversal. Note that for every odd $x \in\{1,2, \ldots, 2 h-1\}, x i$ is a vertex of $D_{i}$.

Since $(2 h-1) i \equiv 0(\bmod 2 \ell-1),(2 h-1) i$ is a common multiple of integers $2 \ell-1$ and $i$, and hence there exists an integer $\alpha \geq 1$ such that

$$
(2 h-1) i=\alpha \operatorname{lcm}(2 \ell-1, i)=\left(\alpha \cdot \frac{2 \ell-1}{\operatorname{gcd}(2 \ell-1, i)}\right) i .
$$

The last equality follows from the fact that $(2 \ell-1) i=\operatorname{gcd}(2 \ell-1, i) \operatorname{lcm}(2 \ell-1, i)$. By Equation 4.1, $2 h-1=\alpha \cdot \frac{2 \ell-1}{\operatorname{gcd}(2 \ell-1, i)}$. Therefore, $\alpha$ is an odd integer because $\frac{2 \ell-1}{\operatorname{gcd}(2 \ell-1, i)}$ is an integer and $2 h-1$ is odd.

We claim that $\alpha=1$. For a contradiction, assume that $\alpha$ is an odd integer greater than 1 . Then, by Equation $4.1,(2 h-1) i-(\alpha-1) \operatorname{lcm}(2 \ell-1, i)=\operatorname{lcm}(2 \ell-1, i)$ and hence

$$
2 h-1-(\alpha-1) \cdot \frac{2 \ell-1}{\operatorname{gcd}(2 \ell-1, i)}=\frac{\operatorname{lcm}(2 \ell-1, i)}{i} .
$$

Since $\alpha-1$ is a possitive even integer, the left side of Equation 4.2 is an odd integer less than $2 h-1$. Moreover, recall that $2 h-1=\alpha \cdot \frac{2 \ell-1}{\operatorname{gcd}(2 \ell-1, i)}$. So, the left side of Equation 4.2 is a positive odd integer less than $2 h-1$. Hence, $\left(2 h-1-(\alpha-1) \cdot \frac{2 \ell-1}{\operatorname{gcd}(2 \ell-1, i)}\right) i$ is an integer in the subsequence $i, 3 i, 5 i, \ldots$, $(2 h-3) i$, and is a multiple of $2 \ell-1$ by Equation 4.2 . However, this implies that vertex 0 of $D_{i}$ is in the subsequence $i, 3 i, 5 i, \ldots,(2 h-3) i$, a contradiction. Thus, the claim holds.

By the claim, $2 h-1=\frac{2 \ell-1}{\operatorname{gcd}(2 \ell-1, i)}$ and so the length of $D_{i}$ is $2 h=\frac{2 \ell-1}{\operatorname{gcd}(2 \ell-1, i)}+1$.

Corollary 4.8 If $\operatorname{gcd}(2 \ell-1, i)=1$, then $D_{i}$ is a tour of $H_{i}$.

We next show that if $D_{i}$ is not a tour of $H_{i}$, then $D_{i}$ is the shortest cycle in $H_{i}$.

Lemma 4.9 Fix an $i$ such that $1 \leq i \leq 2 \ell-2$ and $\operatorname{gcd}(2 \ell-1, i) \neq 1$. Then, each cycle of $H_{i}$ other than $D_{i}$ is of length $\frac{2(2 \ell-1)}{\operatorname{gcd}(2 \ell-1, i)}$.

Proof. Fix a cycle $D$ of $H_{i}$ other than $D_{i}$. Let $2 h$ be the length of $D$. Consider an arbitrary vertex $j$ of $D$. As in the proof of Lemma 4.7, a traversal of $D$ started at vertex $j$ and ended at vertex $-j$ produces the following ordering of the vertices of $D$ :

$$
j,-j+2 i, j-2 i,-j+4 i, j-4 i,-j+6 i, \ldots, j-2(h-1) i,-j+2 h i
$$

where $-j+2 h i \equiv-j(\bmod 2 \ell-1)$. Note that for every even $x \in\{2,3, \ldots, 2 h\},-j+x i$ is a vertex of $D$.

Since $2 h i \equiv 0(\bmod 2 \ell-1), 2 h i$ is a common multiple of integers $2 \ell-1$ and $i$, hence there exists an integer $\alpha \geq 1$ such that

$$
2 h i=\alpha \operatorname{lcm}(2 \ell-1, i)=\left(\alpha \cdot \frac{2 \ell-1}{\operatorname{gcd}(2 \ell-1, i)}\right) i .
$$

By Equation $4.3,2 h=\alpha \cdot \frac{2 \ell-1}{\operatorname{gcd}(2 \ell-1, i)}$. Therefore, $\alpha$ is an even integer.

We claim that $\alpha=2$. For a contradiction, assume that $\alpha$ is an even number greater than 2 . Then, by Equation 4.3, $2 h i-(\alpha-2) \operatorname{lcm}(2 \ell-1, i)=2 \operatorname{lcm}(2 \ell-1, i)$ and hence

$$
2 h-(\alpha-2) \cdot \frac{2 \ell-1}{\operatorname{gcd}(2 \ell-1, i)}=\frac{2 \operatorname{lcm}(2 \ell-1, i)}{i} .
$$


Since $\alpha-2$ is a possitive even integer, the left side of Equation 4.4 is an even integer less than $2 h$. Moreover, recall that $2 h=\alpha \cdot \frac{2 \ell-1}{\operatorname{gcd}(2 \ell-1, i)}$. So, the left side of Equation 4.4 is a positive even integer less than $2 h$. Hence, $-j+\left(2 h-(\alpha-2) \cdot \frac{2 \ell-1}{\operatorname{gcd}(2 \ell-1, i)}\right) i$ is an integer in the subsequence $-j+2 i,-j+4 i$, $\ldots,-j+2(h-1) i$, and is congruent to $-j$ modulo $2 \ell-1$ by Equation 4.4. However, this implies that vertex $-j$ of $D_{i}$ is in the subsequence $-j+2 i,-j+4 i, \ldots,-j+2(h-1) i$, a contradiction. Thus, the claim holds.

By the claim, $2 h=\frac{2(2 \ell-1)}{\operatorname{gcd}(2 \ell-1, i)}$ and so the length of $D_{i}$ is $2 h=\frac{2(2 \ell-1)}{\operatorname{gcd}(2 \ell-1, i)}$.

Corollary 4.10 For every $i \in\{1,2, \ldots, 2 \ell-2\}, D_{i}$ is the shortest cycle in $H_{i}$.

Proof. Fix an $i \in\{1,2, \ldots, 2 \ell-2\}$. If $\operatorname{gcd}(2 \ell-1, i)=1$, then $D_{i}$ is the unique cycle (and hence the shortest cycle) in $H_{i}$ by Corollary 4.8. Otherwise, by Lemmas 4.7 and $4.9, D_{i}$ is shorter than the other cycles in $H_{i}$.

Now, we are ready to prove Lemma 4.5:

Proof of Lemma 4.5: Fix a natural number $q$. By Corollary 4.10, it suffices to show that there are at most $q^{2}-q$ integers $i \in\{1,2, \ldots, 2 \ell-2\}$ such that $D_{i}$ is of length at most $2 q$.

Consider a natural number $p \leq q$. For each $i \in\{1,2, \ldots, 2 \ell-2\}$, if the length of $D_{i}$ is exactly $2 p$, then by Lemma $4.7, \frac{2 \ell-1}{\operatorname{gcd}(2 \ell-1, i)}+1=2 p$ and so

$$
\operatorname{gcd}(2 \ell-1, i)=\frac{2 \ell-1}{2 p-1}
$$

Since each integer $i$ satisfying the above equality has to be a multiple of $\frac{2 \ell-1}{2 p-1}$, there can be at most $2 p-2$ such integers in $\{1,2, \ldots, 2 \ell-2\}$.

Hence, there can be at most $\sum_{p=1}^{q}(2 p-2)=q^{2}-q$ integers $i \in\{1,2, \ldots, 2 \ell-2\}$ such that $H_{i}$ has a cycle of length at most $2 q$.

\section{References}

[1] A. I. Barvinok, D. S. Johnson, G. J. Woeginger, and R. Woodroofe. Finding Maximum Length Tours under Polyhedral Norms. Proceedings of the Sixth International Conference on Integer Programming and Combinatorial Optimization (IPCO), Lecture Notes in Computer Science, 1412 (1998) 195-201.

[2] Z.-Z. Chen and L. Wang. An Improved Randomized Approximation Algorithm for Max TSP. Submitted.

[3] Z.-Z. Chen, Y. Okamoto, and L. Wang. Improved Deterministic Approximation Algorithms for Max TSP. To appear in Information Processing Letters.

[4] R. Hassin and S. Rubinstein. A 7/8-Approximation Approximations for Metric Max TSP. Information Processing Letters, 81 (2002) 247-251.

[5] H. Kaplan, M. Lewenstein, N. Shafrir, and M. Sviridenko. Approximation Algorithms for Asymmetric TSP by Decomposing Directed Regular Multigraphs. Proceedings of the 44th Annual IEEE Symposium on Foundations of Computer Science, pp. 56-75, 2003.

[6] R. M. Karp, E. Upfal, and A. Wigderson. Constructing a Perfect Matching is in random NC. Combinatorica, 6 (1986) 35-48.

[7] A. V. Kostochka and A. I. Serdyukov. Polynomial Algorithms with the Estimates $\frac{3}{4}$ and $\frac{5}{6}$ for the Traveling Salesman Problem of Maximum (in Russian). Upravlyaemye Sistemy, 26 (1985) 55-59.

[8] K. Mulmuley, U. V. Vazirani, and V. V. Vazirani. Matching is as easy as matrix inversion. Combinatorica, 7 (1987) 105-113. 